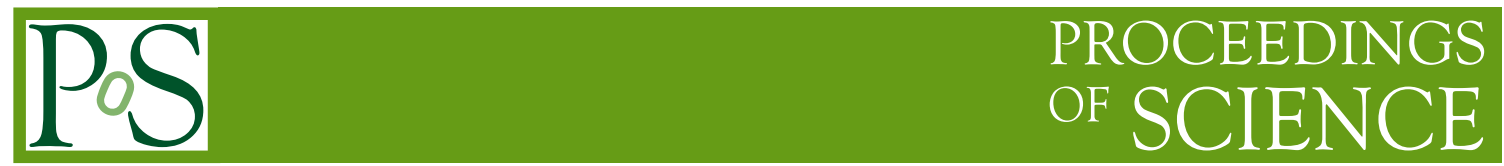

\title{
Polarimetry at the AGS
}

\author{
A. Poblaguev*; E. Aschenauer, G. Atoian, A. Basilevsky, K. O. Eyser, H. Huang, \\ Y. Makdisi, W. Schmidke, D. Smirnov, K. Yip, A. Zelenski \\ Brookhaven National Laboratory, Upton, NY, 11973
}

\section{Alekseev, D. Svirida}

Institute for Theoretical and Experimental Physics,Moscow, 117218, Russia

\begin{abstract}
Proton beam polarization measurement is essential for the Relativistic Heavy-Ion Collider (RHIC) spin program at Brookhaven National Laboratory (BNL). A proton-Carbon polarimeter is employed to monitor beam polarization at the Alternating Gradient Synchrotron (AGS) which injects $24 \mathrm{GeV} / \mathrm{c}$ protons into the RHIC rings. The methods of the polarization measurements and systematic errors are discussed. The results of the experimental testing of the "zero emittance polarization" model is given.
\end{abstract}

XVth International Workshop on Polarized Sources, Targets, and Polarimetry, September 9-13, 2013

Charlottesville, Virginia, USA

\footnotetext{
*Speaker.

†E-mail: poblaguevebnl.gov
} 


\section{Introduction}

The RHIC accelerator complex [1, 2] provides high energy high intensity polarized proton colliding beams for the experiments. The complex consists of several accelerators including the AGS, which accelerates protons from 2.3 to $23.6 \mathrm{GeV} / \mathrm{c}$ for injection to the RHIC rings. Since the polarization losses at depolarizing resonances is a well known problem for acceleration of polarized beams, monitoring the AGS beam polarization is an important requirement for optimization of the RHIC performance.

\section{AGS p-Carbon CNI Polarimeter}

The AGS p-Carbon Coulomb Nuclear Interference (CNI) polarimeter is based on proton carbon elastic scattering with low momentum transfer (the CNI region) and measurement of asymmetry in recoil carbon nuclei production [3]. Schematically, the polarimeter consists of 8 detectors, 12 silicon strips each, and a very thin carbon target as shown on Fig. 1. We use Hamamatsu single strip PIN photodiodes in 45-degree detectors $(1,4,5,8)$. 90-degree detectors consist of Si strips manufactured at BNL. Upper $(2,7)$ and lower $(3,6) 90$-degree detectors use silicon strips of different width, $1 \mathrm{~mm}$ and $2 \mathrm{~mm}$, respectively.

Very thin $(27 \mathrm{~nm})$ carbon foils are used as a target. In the RHIC Run13 we used 50 and 125 $\mu \mathrm{m}$ wide foils in vertical targets and 75 and $125 \mu \mathrm{m}$ wide horizontal targets. The distance from the target to the detectors is equal to 30 and $51 \mathrm{~cm}$ for 90- and 45-degree detectors, respectively.

Three main types of measurements provided by the AGS polarimeter are illustrated in Fig. E:

- Fixed target measurements. In every AGS cycle the target is put to the center of the beam. The beam polarization is measured at flattop, i.e. after acceleration to the $24 \mathrm{GeV} / \mathrm{c}$. Some rate decreasing (Fig. 2 (left)) during the measurements is attributed to the growing of the beam emittance. A typical measurement (40M events) takes few minutes and provides the statistical accuracy of the measured polarization of about $\delta P \sim 2 \%$.

- Sweep target measurements. During these measurements the target crosses the beam with a constant velocity. The target coordinate $x$ (in units of the intensity profile sigma) may be reconstructed from the rate in the detectors. The polarization profile is assumed as Gaussian

$$
P(x)=P_{\max } e^{-R x^{2} / 2}
$$
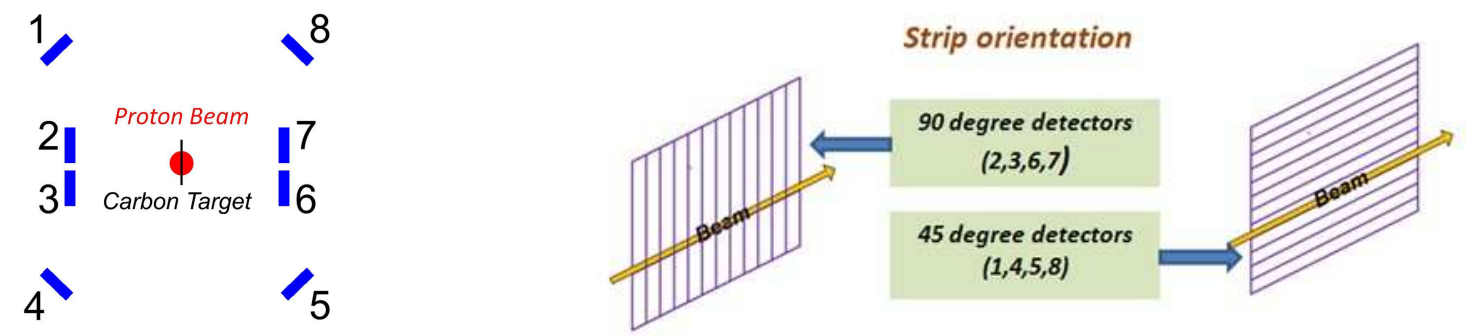

Figure 1: A schematic view on the AGS p-Carbon polarimeter. 

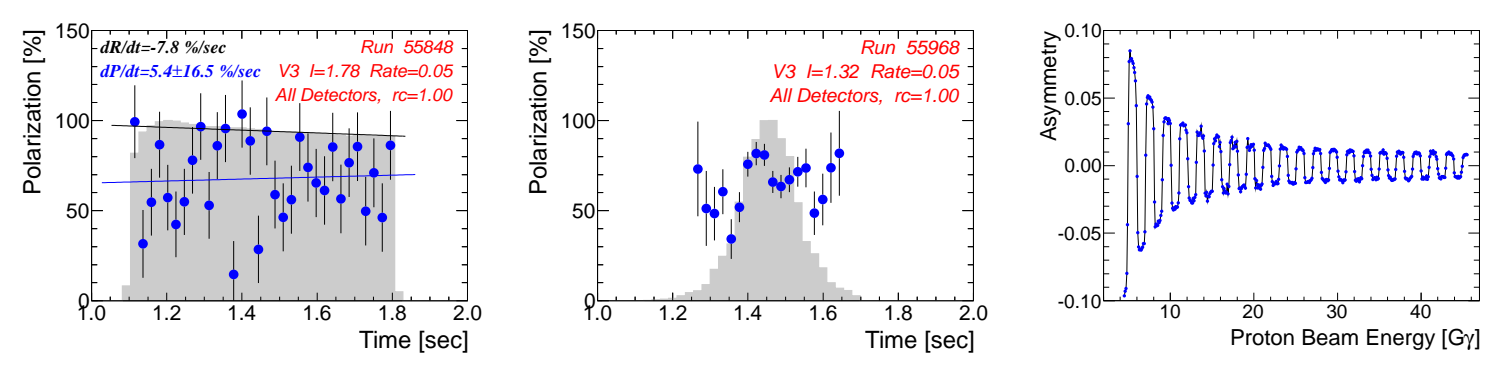

Figure 2: Polarization measurements in the AGS p-Carbon polarimeter: fixed target (left), sweep target (center), and ramp measurements (right). Filled histograms (left and center) display the rate (a.u.) in the detectors which is proportional to the beam intensity at the target location. The time is counted relative to the beginning of the AGS cycle.

The $P_{\max } \sim 70 \%$ is actually a polarization determined in the fixed target measurements and the squared ratio of intensity and polarization profiles $R=\left(\sigma_{\text {int }} / \sigma_{\text {pol }}\right)^{2} \sim 0.1$ is commonly used to parametrize the polarization profile. Since the data taking in the sweep target measurements is less efficient than in the fixed target measurements, it takes a longer time to measure the polarization profile $R$. The average beam polarization may be calculated as

$$
\langle P\rangle=P_{\max } / \sqrt{1+R}
$$

- Ramp measurements. Ramp measurements are fixed target measurements taken during the proton beam acceleration. The AGS "Siberian snakes" flip the proton spin polarity at integer values of the $G \gamma$ [1] as shown on Fig. 2 (right). The asymmetry reduction during the measurements is partially due to the dependence of analyzing power $A_{N}(t)$ on the proton beam energy.

\section{Polarization measurement}

The WFD based Data Acquisition system (DAQ) triggers signals above the kinetic energy threshold of about $300 \mathrm{keV}$ and within a configured time window relative to the beam scattering on the target (Fig. 3). Signal amplitude $A$ and time $t$ are calculated in the WFD firmware. Three parameters, time offset $t_{0}$, thickness of the Si detector dead-layer $x_{D L}$ and the ADC gain $\alpha$, which are determined in a calibration, allow us to reconstruct the kinetic energy $T\left(\alpha A, x_{D L}\right)$ and time of flight $t-t_{0}$. The recoil Carbons are identified by comparison of the $T$ and $t-t_{0}$. For the polarization measurements, the Carbon kinetic energy is selected within a 400-900 keV range.

The AGS polarization $P$ is mostly vertical. Such a polarization can be determined by measurement of the asymmetry $a$ of the left/right production of recoil Carbons

$$
a=\left\langle A_{N}\right\rangle P .
$$

The average analyzing power $\left\langle A_{N}\right\rangle$ is calculated using the measured energy $T$ of the Carbon and theoretical dependence of the $A_{N}(t)$ (see Fig. B (left)) on momentum transfer $t=-2 M_{C} T$. The 

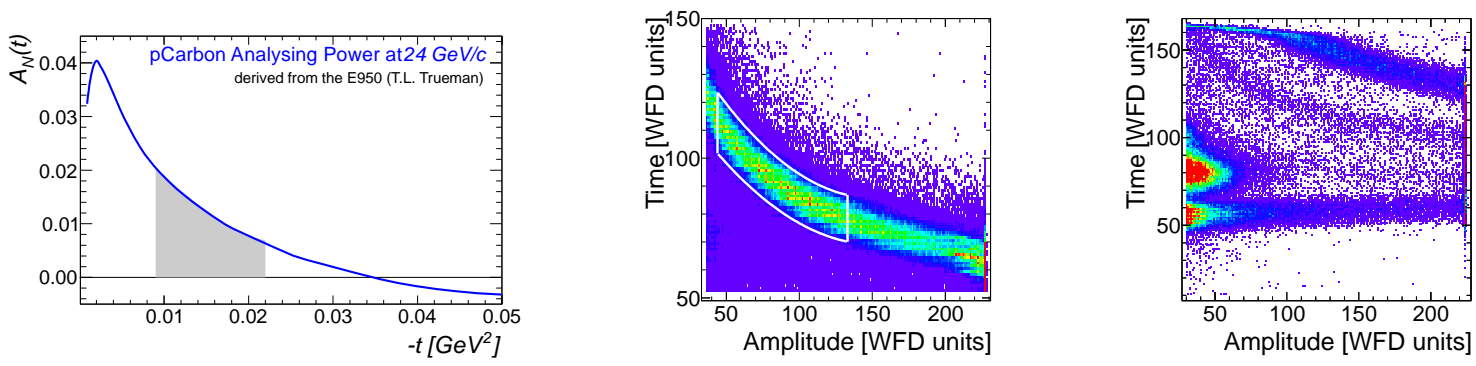

Figure 3: The p-Carbon analyzing power used in data analysis (left). The filled area highlights the kinetic energy range for the polarization measurements. A typical events distribution in one Si strip accepted by the DAQ (center). The width of the $t(A)$ dependence is defined by the beam bunch length. The white line contour shows the event selection for the polarization measurement. A time versus amplitude dependence in measurement with shifted event time window (right). In a regular measurement the high rate induced pulse $(A \lesssim 50)$ and prompt (i.e. fast protons and/or pions penetrating through the Si strips) are cut off by the DAQ.
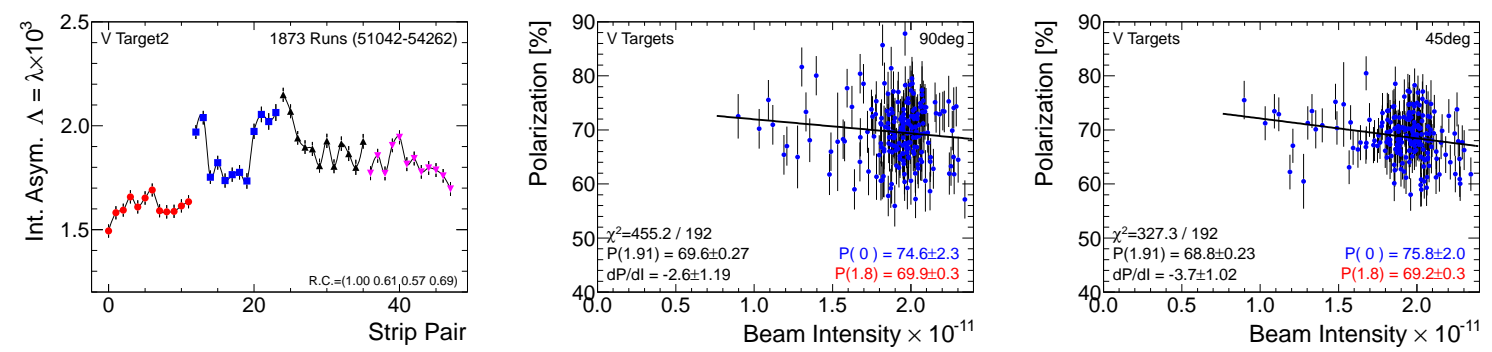

Figure 4: Intensity asymmetry measured independently by 48 pairs of the Si strips (left). Comparison of polarization measured by 90 -degree (center) and 45-degree (right) detectors.

beam spin flipping during the measurements allows one to strongly suppress the systematic errors caused by uncertainties in the acceptance $\varepsilon$ and/or intensity $\lambda$ asymmetries:

$$
a=\frac{\sqrt{N_{R}^{\uparrow} N_{L}^{\downarrow}}-\sqrt{N_{L}^{\uparrow} N_{R}^{\downarrow}}}{\sqrt{N_{R}^{\uparrow} N_{L}^{\downarrow}}+\sqrt{N_{L}^{\uparrow} N_{R}^{\downarrow}}}
$$

Here, $N_{L R}^{\uparrow \downarrow}$ are numbers of detected events depending on the spin polarity and left/right position of the detectors. The $\varepsilon$ and $\lambda$ are calculated using the similar formulas. The Eq. (3.2) usually referred as square root formula gives the exact, i.e. the systmatic error free, solution for the $a$ if variations of asymmetries $a, \varepsilon$, and $\lambda$ during the measurements are not correlated.

A detailed analysis of the square root formula shows that the calculated value of $\lambda$ is modified by the systematic errors in evaluation of the $\left\langle A_{N}\right\rangle$. The distribution of the intensity asymmetry $\lambda$ (averaged over the RHIC Run12 data) measured independently by 48 pairs of Si strips (Fig. $\emptyset$ (left)) should be interpreted as indication of the 1-2\% fluctuations of the calculated analyzing power $\left\langle A_{N}\right\rangle$ due to the systematic errors. Even though the square root formula provides unbiased (with high accuracy) estimate of the polarization asymmetry $a$, the systematic errors in calculation of $\left\langle A_{N}\right\rangle$ are propagated to the measured values of the polarization $P$. Comparison of the polarization measured 
by 90 -degree and 45 -degree detectors (Fig. đ) indicates a $1 \%$ discrepancy which may be attributed to the discussed systematic errors.

\section{Systematic errors}

There are several possible sources of systematic errors in the calculation of the analyzing power:

Energy losses in the target. Due to the energy losses in the target, the measured kinetic energy of the Carbon is smaller than energy acquired at the proton scattering. According to the numerical estimates, the measured polarization is underestimated by about $\delta P / P \sim 1 \%$ due to the dependence of the analyzing power on recoil Carbon energy.

Background. Generally, the theoretical analyzing power is not applicable to the background events. A study based on variation of the cuts for event selection shows that background dependent systematic error does not exceed $1 \%$.

Energy calibration. Due to the dependence of analyzing power $A_{N}(t)$ on recoil Carbon energy, a $5 \%$ error in the energy scale will result in approximately 5\% error in measured value of the polarization.

The calibration of the AGS polarimeter includes (i) determination of the gain $\alpha$ in special runs in which $\mathrm{Si}$ detectors are exposed by $5.486 \mathrm{MeV} \alpha$-particles emitted by ${ }^{241} \mathrm{Am}$ source and (ii) determination of the dead-layer $x_{D L}$ and time offset $t_{0}$ from the analysis of the measured time dependence on the signal amplitude (see Fig. 3 (center)). The difficulties of such a calibration are obvious: the $\alpha$ energy is much larger then Carbon energy and the determination of $x_{D L}$ and $t_{0}$ is very sensitive to a small variation of the dead-layer model. As a result this calibration can not be considered as reliable.

Recently, another calibration method was tested. Due to the Si stopping power dependence on the proton energy, the measured time for the prompt events depends on signal amplitude as $t=t_{0}+c A^{0.6}$. Such a dependence can be easily recognized in the fit to the data. The measured value of the factor $c$ can be compared with a calculated one (depending on the Si thickness) to verify the results the measurements. The tests gave an optimistic results for some Si strips. However, the problem caused by interference of the prompt and induced pulse signals is not resolved yet.

Theoretical analyzing power $\mathbf{A}_{\mathbf{N}}(\mathbf{t})$. In the data analysis we use the analyzing power which is a theoretical extrapolation of the E950 experimental data at $21 \mathrm{GeV} / \mathrm{c}$ [ 㺻. Potentially, the analyzing power can be measured by the AGS polarimeter data. ${ }^{1}$ Comparison of the measured and theoretical analyzing powers in Fig. 5 shows a significant discrepancy. An issue with energy calibration discussed above does not allow us to resolve this discrepancy. For this reason the AGS polarimeter should be used for relative polarization measurements only.

\section{Rate corrections}

Since the DAQ may accept only one signal per Si strip per bunch, the signal detection efficiency is rate dependent

$$
\varepsilon(r)=1-k r
$$

\footnotetext{
${ }^{1} \mathrm{Up}$ to some scaling factor unless the exact beam polarization is known.
} 


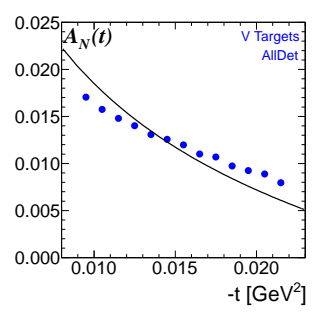

Figure 5: Comparison of theoretical (solid line) and measured (blue dots) analyzing power. The measured $A_{N}(t)$ is scaled to give the same average beam polarization as the theoretical one.

where $r$ is rate per bunch in a strip and $k$ is a rate correction factor. Generally, $k$ depends on the ratio $\rho \approx 0.5$ of good, i.e. selected for polarization measurement, to total number of triggered events (see Fig. (6), time/amplitude distributions of the good and background events, and the algorithm of the signal reconstruction in the WFD firmware. Roughly, it may be estimated as $k=1-\rho / 2 \approx 0.75$. Inefficiency of the signal detection results in the systematic error in the measured polarization.

$$
P_{\text {meas }}=P_{\text {beam }} \frac{1-2 k r}{1-k r} \approx(1-k r) P_{\text {beam }}
$$

Depending on the beam intensity and target width, the rate per strip may be as large as $r \sim 0.15$ which results in a significant bias of the measured polarization unless the rate corrections are applied.

Three different methods of the experimental evaluation of the value of $k$ were studied.

(1) All Si detectors measure the same beam polarization. In particular, the dependence of measured polarization on the beam intensity, $d P / d I$, should be the same for any group of detectors. The value of $d P / d I$ is sensitive to the rate correction parametrization. Due to the orientation of the 90degree detectors, the rates in these Si strips are systematically different. This may be employed for determination of the actual average value of the $d P / d I$. After that, the parameter $k$ may be evaluated for the 45 degree detectors. The main drawback of this method is big statistical errors. Even for the complete RHIC Run data which contains several thousand of polarization measurements, the statistical error of determination $k$ is about $\delta k \sim 0.1 \div 0.2$.
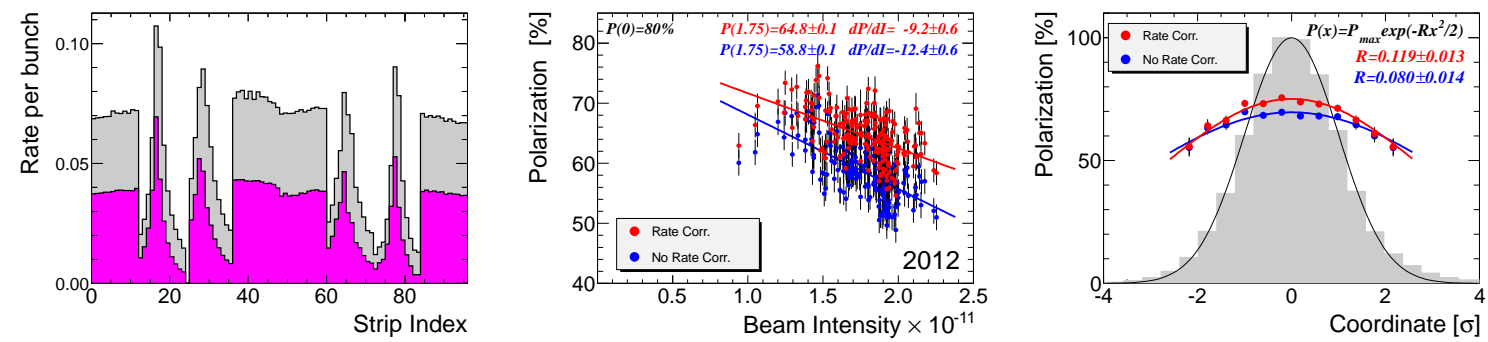

Figure 6: Rate per bunch in the Si strips (left). The rate for good events only is highlighted using magenta color. The polarization versus beam intensity (center) and profile measurements (right) depending on the rate correction. The results of measurements with/without rate corrections are shown using red/blue colors respectively. 


\begin{tabular}{cccccc} 
Target & Intesity & $\mathbf{P}_{\max }$ & $\mathbf{R}$ & $\mathbf{P}_{0}$ & $\mathbf{P}_{\text {source }}$ \\
\hline V3 & 2.06 & $70.9 \pm 0.5$ & $0.098 \pm 0.010$ & $81.2 \pm 1.1$ & $81.2 \pm 0.1$ \\
V3 & 1.51 & $74.2 \pm 0.5$ & $0.113 \pm 0.010$ & $85.5 \pm 1.3$ & $82.0 \pm 0.1$ \\
V3 & 1.05 & $74.7 \pm 0.8$ & $0.049 \pm 0.015$ & $80.1 \pm 1.6$ & $81.6 \pm 0.1$ \\
\hline H3 & 2.09 & $67.2 \pm 0.6$ & $0.103 \pm 0.014$ & $77.5 \pm 1.0$ & $81.0 \pm 0.1$ \\
H3 & 1.50 & $72.3 \pm 0.8$ & $0.093 \pm 0.017$ & $84.1 \pm 1.3$ & $82.2 \pm 0.1$ \\
H3 & 1.08 & $73.2 \pm 0.9$ & $0.047 \pm 0.016$ & $78.6 \pm 1.6$ & $81.7 \pm 0.1$ \\
\hline V3 & 1.91 & $69.0 \pm 0.2$ & Fixed target & $79.7 \pm 1.0$ & $81.1 \pm 0.1$
\end{tabular}

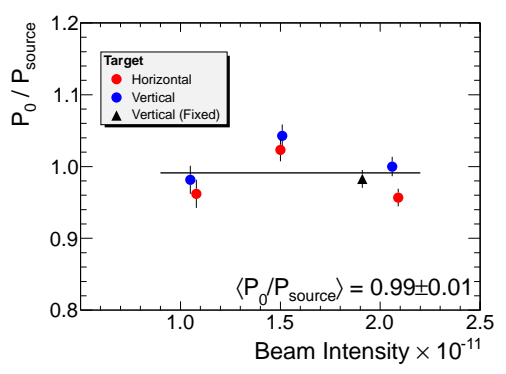

Figure 7: Comparison of the zero emittance polarization, measured in the RHIC Run13 with the source polarization.

(2) The acceptance asymmetry $\varepsilon$ is affected by rate corrections in the same way as the polarization asymmetry $a$. Since the statistical errors of determination of the $\varepsilon$ and $a$ are the same, but the value of $\varepsilon \sim 0.05$ appeared to be much larger than $a \sim 0.01$, the variations of $\varepsilon$ in the polarization profile (sweep target) measurements are sensitive to the parameter $k$. This method employs the linear dependence of the $\varepsilon$ on the target coordinate. Only a few such measurements allow us to reach statistical accuracy better than $\delta k \lesssim 0.1$. However, the method is sensitive to the boundary conditions in the event selection and the results are not stable.

(3) The efficiency of the detection of any event in the Si strip is defined by Poisson statistics $\left(1-e^{-r}\right) / r$. The probability that this event is a good one may be evaluated by studying the good to total event ratio $\rho(r)=\rho_{0}(1-\kappa r)$ in the profile measurement. The efficiency (5.1) of a good event detection in a Si strip may be calculated as

$$
\varepsilon(r)=1-k r=(1-\kappa r) \times\left(1-e^{-r}\right) / r \approx 1-(\kappa+0.5) r \Rightarrow k=\kappa+0.5
$$

Even a single profile measurement provides a good statistical accuracy of determination of the $k$ in every Si strip. However, systematic errors due to threshold effects in the event selection are not well understood at the moment.

Neither of these methods may be currently considered as a reliable one for the determination of the rate correction factor $k$. Nonetheless, a comparison of these methods gives us a confidence that rate correction factor is controlled with an accuracy of about $\delta k \sim 0.1$. As a result, the error in determination of the polarization due to the inaccuracy of the rate corrections does not exceed $1-2 \%$.

\section{Zero emittance polarization}

If the development of a polarization profile is the primary reason for the reduction of the average polarization $\langle P\rangle$ then [5]

$$
\langle P\rangle=\frac{P_{0}}{\left(1+R_{x}\right)\left(1+R_{y}\right)}
$$

where $P_{0}$ is zero emittance polarization and $R_{x, y}$ are vertical and horizontal polarization profiles (2.1). This model is expected to be valid at the AGS. The $P_{0}$ may be related to the source polariza- 
tion $P_{\text {source, }}$, measured by the $200 \mathrm{MeV}$ absolute polarimeter [6] with a $1 \%$ systematic error

$$
P_{0} / P_{\text {source }} \approx 0.985
$$

Here we accounted the effective polarization loss caused by a non-vertical spin direction at the injection to the AGS and at the flattop.

The profile measurements allows us to make an experimental estimate

$$
P_{0}^{(\text {meas })}=P_{\max }^{(x)} \sqrt{1+R_{x}}\left(1+R_{y}\right)=P_{\max }^{(y)} \sqrt{1+R_{y}}\left(1+R_{x}\right)
$$

In the RHIC Run13, the polarization profiles, both vertical and horizontal, were measured for several beam intensities. Results of these measurements shown on Fig. 7 may be summarized as

$$
P_{0}^{\text {(meas) }} / P_{\text {source }}=0.99 \pm 0.01
$$

A perfect agreement with (5.2) should be considered as unexpected one if we take into account the issues with energy calibration and discrepancy between the measured and theoretical analyzing power $A_{N}(t)$. Nonetheless, we may conclude that polarization measured by the AGS p-Carbon polarimeter is in good agreement with a few percent accuracy with the actual beam polarization.

A verification of the zero emittance polarization model may allow us to properly normalize the analyzing power measured by the AGS p-Carbon polarimeter.

\section{Summary}

The AGS p-Carbon polarimeter provides fast and reliable relative polarization measurements. It is employed for monitoring the polarization delivered to the RHIC rings as well as for numerous developments of the polarized beam at the AGS. The statistical accuracy of the polarization measurement in a single run is about $\delta P \sim 2 \%$ The systematic accuracy of the relative measurement of polarization is about $\delta P \lesssim 2 \%$ We may expect that the polarimeter measures absolute polarization with a few percent accuracy. The AGS p-Carbon polarimeter may be used as absolute polarimeter if (i) the conservation of the zero emittance polarization will be proved and (ii) the energy calibration problem will be be resolved.

\section{Acknowledgments}

This work is performed under Brookhaven Science Associates, LLC, contract No. DE-AC0298CH10886 with the U.S. Department of Energy. Funding is also provided from the RIKEN BNL Research Center.

\section{References}

[1] T. Roser, AIP Conf. Proc., 980, p. 15 (2008).

[2] I. Alekseev et al. NIM A499 (2003) 392.

[3] I. Nakagava et al. AIP Conf. Proc. 980 (2008) 308.

[4] T. L. Trueman, hep-ph/0305085.

[5] W. Fischer and A. Bazilevsky, Phys.Rev.ST Accel.Beams 15 (2012) 041001.

[6] A. Zelenski, et al., J. Phys. Conf. Ser. 295, 012132 (2011). 